\title{
EFEITO DO PLASMA RICO EM GLICOSE NA APOPTOSE DE NEUTRÓFILOS DE CÃES
}

\author{
Maria Carolina Ramos Nogueira1, Priscila Preve Pereira ${ }^{1}$, Breno Fernando Martins \\ Almeida', Anelise Maria Bosco ${ }^{1}$, Tatiana Sousa Barbosa², Paulo César Ciarlini' \\ 1 UNESP - Campus Araçatuba \\ 2 UNESP - Campus Botucatu \\ Correspondência: Paulo César Ciarlini: ciarlini@fmva.unesp.br
}

\begin{abstract}
RESUMO: A hiperglicemia é considerada a principal causa do aumento da mortalidade em pacientes diabéticos humanos, por promover a aceleração da apoptose dos neutrófilos, essenciais para o funcionamento do sistema imune. Desta forma, dá-se um efeito imunossupressor que contribui para o aumento da susceptibilidade dos pacientes a infecções bacterianas graves. Na espécie canina não há estudos semelhantes quanto ao efeito imunossupressor da hiperglicemia, de modo que se testou a hipótese de que o excesso de glicose acelera a apoptose dos neutrófilos em cães. Para tal, substituiu-se o plasma de amostras de sangue de 10 cães sadios por plasma autólogo, homólogo normoglicêmico e homólogo hiperglicêmico. Todas as amostras foram incubadas por duas e quatro horas. As mensurações de glicose foram realizadas pelo método cinético glicose oxidase e o índice apoptótico dos neutrófilos calculado pelo método morfométrico. Em todos os ensaios o índice apoptótico aumentou após duas e quatro horas. Após quatro horas, o índice apoptótico das amostras incubadas com plasma homólogo hiperglicêmico foi significativamente superior $(p<0,05)$ aos das amostras incubadas com homólogo normoglicêmico e autólogo. Concluiu-se que in vitro o plasma hiperglicêmico aumenta a taxa de apoptose dos neutrófilos em cães, fortalecendo a hipótese de que cães portadores de doenças hiperglicemiantes possam igualmente sofrer imunossupressão por disfunções neutrofílica.
\end{abstract}

Palavras-chave: hiperglicemia; imunossupressão; polimorfonuclear

\section{EFFECT OF RICH GLUCOSE PLASMA IN APOPTOSIS OF NEUTROPHILS IN DOGS}

\begin{abstract}
Hyperglycemia is considered the main cause of increased mortality in diabetic humans, to promote the acceleration of neutrophil apoptosis, essential for the functioning of the immune system. Thus, there is an immunosuppressive effect that contributes to the increased susceptibility of patients to serious bacterial infections. In canine species there is no similar studies on the immunosuppressive effect of hyperglycemia, so that it tested the hypothesis that excess glucose accelerates neutrophil apoptosis in dogs. To this end, replaced the plasma of blood samples from 10 healthy dogs plasma autologous, homologous counterpart normoglycemia and hyperglycemia. All samples were incubated for two and four hours. The glucose measurements were performed using kinetic glucose oxidase and neutrophil apoptotic index calculated by the morphometric method. In all tests the apoptotic index increased after two and four hours. After four hours, the apoptotic index of the samples incubated with homologous plasma hyperglycemia was significantly higher $(p<0.05)$ to the samples incubated with homologous and autologous normoglycemia. It was concluded that plasma hyperglycemia in vitro increases the rate of neutrophil apoptosis in dogs, strengthening the hypothesis that dogs with hyperglycemic disease may also suffer immunosuppression neutrophil dysfunction.
\end{abstract}

Key Words: hyperglycemia; immunosuppression; polymorphonuclear 


\section{INTRODUÇÃO}

Os neutrófilos são considerados a primeira linha de defesa do organismo (imunidade inata), movendo-se em direção ao micro-organismo, fagocitando e destruindo-o (Cohen et al., 2001), mas sendo incapaz de um esforço prolongado devido a sua meia vida, in vivo, de aproximadamente 12 horas (Tizard et al., 2002). A meia-vida de neutrófilos pode ser aumentada ou reduzida com a aceleração ou atraso da apoptose (Squier et al., 1995). A apoptose é um mecanismo fisiológico de controle da população normal de neutrófilos (Oguma et al., 2000) e age também durante a inflamação, uma vez que estes possuem a morte geneticamente programada (Majewska et al., 2000, Glowacka et al., 2002).

A hiperglicemia em cães ocorre comumente em condições de estresse e doenças como diabetes mellitus e o hiperadrenocorticismo (Nelson; Couto, 2006). Pacientes que sofrem de tais enfermidades hiperglicemiantes apresentam maior risco de infecção e este fato relaciona-se com defeitos na função microbicida e fagocitária dos neutrófilos (Whyte et al., 1993; McManus et al., 2001).

O excesso de glicose na corrente circulatória também causa alterações no metabolismo energético do neutrófilo (via do poliol), aumentando a produção de sorbitol, induzindo uma diminuição da adesão, quimiotaxia e migração ao foco inflamatório. O sorbitol leva a diminuição da disponibilidade da enzima $\mathrm{NADPH}$, interferindo na interação leucócito-endotélio. A hiperglicemia também causa um aumento na produção de espécies reativas de oxigênio (ERO), diminuição da resistência ao estresse oxidativo e avançada glicosilação protéica (Brownlee, 2001; Alba-Loureiro et al., 2007).
A glutamina é um aminoácido que exerce efeito protetor sobre a função neutrofílica. Esta é transformada em glutationa pelos neutrófilos, 0 que aumenta o seu efeito bactericida através da ação antioxidante, além de estabilizar a função mitocondrial, retardando a ocorrência de apoptose. Em ratos diabéticos, foram observadas alterações nas concentrações de glutamina, o que compromete a resposta inflamatória nestes indivíduos (Pithon-Curi et al., 2003; Alba-Loureiro et al., 2007).

Segundo Tennenberg et al. (1999) - aumento da susceptibilidade e severidade das infecções em pacientes humanos diabéticos deve-se ao fato da hiperglicemia causar a aceleração da apoptose dos neutrófilos. Considerandose que a hiperglicemia não é o único fator pró-apoptótico em pacientes diabéticos, protocolos foram desenvolvidos para se avaliar o efeito isolado de diferentes concentrações de glicose sobre a apoptose dos neutrófilos. Neste sentido, estudo realizado "in vitro" por Turina et al. (2006) revelou que a exposição de neutrófilos humanos a altas concentrações de glicose plasmática $(10 \mathrm{mg} / \mathrm{dL}, \quad 100 \mathrm{mg} / \mathrm{dL}, \quad 200 \mathrm{mg} / \mathrm{dL} \quad$ e $400 \mathrm{mg} / \mathrm{dL}$ ) no período de meia e vinte quatro horas não promove aumento significativo da apoptose. Tais divergências entre essas pesquisas reforçam a necessidade de mais estudos uma vez que ainda não está clara a relação entre a hiperglicemia, a apoptose e disfunção dos neutrófilos.

Considerando que são diversos os mecanismos que afetam a função neutrofílica de pacientes portadores de doenças hiperglicêmicas in vivo, decidiu-se desenvolver um protocolo que permita avaliar o efeito isolado de plasma enriquecido com glicose sobre a taxa de apoptose dos neutrófilos de cães sadios a fim de testar in vitro a hipótese de que o plasma enriquecido 
com alta concentração de glicose acelera a apoptose de neutrófilos de cães sadios.

\section{MATERIAL E MÉTODOS}

Foram utilizados 10 cães das raças Pastor Alemão, Labrador, Rottweiler e Golden Retriever, de ambos os sexos, saudáveis, com idade entre três e cinco anos provindos do canil da Polícia Militar do Município de Araçatuba. Os animais apresentaram hemograma e glicemia normais, não apresentaram alterações no exame físico geral (Feitosa, 2008), sem recente vacinação ou tratamentos com medicamentos antimicrobianos, antinflamatórios e imunossupressores.

Foi realizada colheita de $10 \mathrm{~mL}$ de sangue em tubos siliconizados heparinizados à vácuo ${ }^{1}$. Todas as amostras foram mantidas refrigeradas até o momento de seu processamento.

O sangue colhido foi submetido à centrifugação a $700 \mathrm{~g}$ durante 15 minutos para a obtenção do plasma. $\mathrm{O}$ "pool" de plasma homólogo normoglicêmico foi produzido a partir da retirada do plasma dos animais e armazenado em tubos de polipropileno a $-20^{\circ} \mathrm{C}$ até o momento do uso sem o acréscimo de nenhuma substância. Para formar o "pool" de plasma homólogo hiperglicêmico cada um $\mathrm{ml}$ de plasma foi acrescido $5 \mathrm{mg}$ de glicose para atingir uma concentração final de aproximadamente $13,83 \mathrm{mmol} / \mathrm{L}(248,77$ $\mathrm{mg} / \mathrm{dL}$ ).

Para a avaliação do efeito do plasma rico em glicose sobre a apoptose dos neutrófilos o sangue total de 10 cães foi submetido a quatro diferentes ensaios. Para tal, quatro alíquotas de $500 \mu \mathrm{L}$ de sangue total foram centrifugadas ( $700 \mathrm{~g} / 15$ minutos) e $100 \mu$ do sobrenadante substituído por um volume equivalente de plasma

\footnotetext{
${ }^{1}$ Vacutainer plus plastic Heparin, Cod. 367993, Becton-Dickson, New Jersey, USA.
}

homólogo normoglicêmico (Tubo 1), homólogo hiperglicêmico (Tubo 2) e plasma autólogo (Tubo 3). Foram retirados $500 \mu \mathrm{L}$ de sangue para obtenção de plasma e conseguinte determinação da concentração de glicose após a adição dos plasmas homólogos normoglicêmicos e hiperglicêmicos.

Em cada momento foram realizados dois esfregaços por amostra tingidos com corante panótico rápido comercial $^{2}$. Para a determinação do índice apoptótico, um número mínimo de 100 neutrófilos foram analisados morfologicamente por microscopia óptica em aumento de 1.000X, conforme protocolo descrito por Campos et al. (2004) e Batista et al. (2005). Para a quantificação das células apoptóticas, foram contadas somente as que apresentaram pelo menos três das seguintes características morfológicas peculiares do processo: condensação do citoplasma (intensa coloração citoplasmática), condensação nuclear (compactação da cromatina nuclear em massas densas e uniformes, alinhadas no lado interno da membrana nuclear), fragmentação nuclear (convolução e fragmentação da membrana nuclear sem cariorrexe ou ruptura), fragmentação celular (formação de corpos apoptóticos). Os corpos apoptóticos em grande número e próximos uns dos outros foram quantificados como o resultado de uma única célula em apoptose. Quando distantes entre si, foram considerados como resultados de apoptose em células diferentes e contabilizados como tal.

A fim de não incluir corpos apoptóticos de linfócitos na avaliação em questão, levou-se em consideração as informações descritas por Nagami e colaboradores (2002), em que corpos apoptóticos de linfócitos apresentam

\footnotetext{
${ }^{2}$ Instant-Prov, NEWPROV, Pinhais- PR.
} 
citoplasma basofílico (coloração azulada), menor tamanho e fragmentos nucleares mais irregulares e os de neutrófilos citoplasma neutrofílico e de maior tamanho.

Com auxílio de um programa computacional estatístico ${ }^{3}$ e conforme preconizado por Zar (1984), após os estudos das distribuições das variáveis quanto à normalidade (teste KS) e homocedasticidade (teste Bartlett), para as comparações entre momentos em cada ensaio experimental foi utilizadas a ANOVA e o pós-teste de Tukey.

\section{RESULTADOS E DISCUSSÃO}

As médias de glicose plasmática dos animais após acréscimo de plasma autólogo e homólogo normoglicêmico ficaram dentro da faixa de normalidade para a espécie (Kaneko, 2008), enquanto as amostras acrescidas de plasma homólogo enriquecido com glicose (homólogo hiperglicêmico) foram equivalentes aos de um cão portador de diabetes mellitus (Tabela 1).

Tabela 1- Porcentagem de neutrófilos apoptóticos (Médias \pm desvios padrão) de cães sadios, antes (0h) e após 2 e 4 horas de incubação com plasma autólogo normoglicêmico $(4,44 \pm 0,37 \mathrm{mmol} / \mathrm{L}$ glicose), homólogo normoglicêmico $(4,40 \pm$ $0,29 \mathrm{mmoVL}$ glicose) e hiperglicêmico (13,83 $\pm 1,27 \mathrm{mmo} / \mathrm{L}$ glicose)

\begin{tabular}{l|c|cc}
\hline & Tempo 0h & Tempo 2h & Tempo 4h \\
\hline Plasma & $1,8 \pm 0,87$ & $30,9 \pm 7,48$ & $30,4 \pm 10,64$ \\
Autólogo & $\mathrm{A}^{1} \mathrm{a}^{2}$ & $\mathrm{Ba}$ & $\mathrm{Bb}$ \\
$\begin{array}{l}\text { Normoglicêmico } \\
\text { Plasma }\end{array}$ & $2,1 \pm 1,24$ & $28,9 \pm 9,35$ & $33,1 \pm 11,48$ \\
homólogo & $\mathrm{Aa}$ & $\mathrm{Ba}$ & $\mathrm{Bab}$ \\
normoglicêmico & & & \\
\hline $\begin{array}{l}\text { Plasma } \\
\text { homólogo }\end{array}$ & $2,4 \pm 1,82$ & $31,3 \pm 15,19$ & $43,7 \pm 10,50$ \\
hiperg licêmico & $\mathrm{Aa}$ & $\mathrm{Ba}$ & $\mathrm{Ca}$ \\
: Letras maiús culas distintas na mesma linha indicam diferença significativa (p<0,05).
\end{tabular}

${ }^{2}$ Letras minús culas distintas na mesma coluna indicam diferença significativa $(p<0,05)$.

A taxa de apoptose dos neutrófilos no momento imediatamente após 0 acréscimo dos plasmas autólogo, homólogo normoglicêmico e homólogo hiperglicêmico não diferiram

\footnotetext{
${ }^{3}$ SAS/STA Software, Statistical Analysis System Institute, 1997, USA.
}

significativamente entre si (Tabela 1), garantindo que as amostras sanguíneas selecionadas para 0 estudo não sofreram qualquer efeito indutor de apoptose "in vivo". Estes resultados permitem afirmar que as manipulações realizadas com as amostras nos diferentes ensaios não aceleraram a apoptose dos neutrófilos e sugerem que o aumento da taxa de apoptose está associado com o tempo de incubação e a concentração glicêmica.

O índice apoptótico dos ensaios com plasma autólogo e homólogo normoglicêmico não diferiram em nenhum momento, comprovando que a diferença da composição dos plasmas não contribuiu para aceleração da apoptose (Tabela 1). É razoável afirmar que o aumento da taxa de apoptose nos ensaios autólogo e homólogos normoglicêmicos esteja relacionada com tempo e temperatura de incubação das amostras, conforme anteriormente verificado por Sela et al. (2005).

Diferentemente, amostras incubadas com plasma hiperglicêmico tiveram índice apoptótico maior que as normoglicêmicas, sendo tal diferença apenas significativa após quatro horas de incubação (Tabela 1). Estes resultados comprovam que a hiperglicemia acelera a apoptose dos neutrófilos de cães e que este efeito é dependente do tempo. Anteriormente, Tennenberg et al. (1999) verificaram que em humanos a alta concentração de glicose provoca diminuição da longevidade funcional dos neutrófilos, aumenta a retirada destes do foco inflamatório, possibilitando uma maior susceptibilidade à infecções ou infecções mais severas. A aceleração da apoptose de neutrófilos e o aumento da susceptibilidade a infecções também foi observada em ratos diabéticos (AlbaLoureiro et al., 2007).

Divergindo do presente estudo e demais relatos da literatura, neutrófilos caninos incubados com meio de cultura 
enriquecido com glicose (16 mmol/L) não apresentaram alteração da taxa de apoptose (Bosco et al., 2013) e neutrófilos humanos incubados com altas concentrações de glicose plasmática no período de 30 minutos e 24 horas não resultou no aumento significativo da apoptose (Turina et al., 2006). É provável que tal divergência esteja associada ao fato desses autores terem avaliado o efeito da hiperglicemia sob os neutrófilos isolados e não em sangue total.

Diante dos resultados obtidos e das evidências de que in vivo a hiperglicemia é um fator imunossupressor em humanos (Gupta et al., 2007), torna-se necessário aprofundar a presente investigação para confirmar se o mesmo ocorre em cães e em quais doenças hiperglicemiantes. $O$ protocolo desenvolvido no presente estudo permitiu identificar o efeito da hiperglicemia sobre a apoptose sem necessidade de isolamento ou cultivo em meio artificial, abrindo perspectivas para novos estudos sobre o efeito próapoptótico da hiperglicemia, assim como para testar in vitro novas estratégias de tratamento das doenças hiperglicemiantes.

\section{CONCLUSÃO}

O plasma rico em glicose aumenta a taxa de apoptose dos neutrófilos de cão in vitro, fortalecendo a hipótese de que cães portadores de doenças hiperglicemiantes possam igualmente sofrer imunossupressão devido à disfunção neutrofílica.

\section{AGRADECIMENTOS}

A FAPESP pela bolsa auxílio (Processo 2009/50315-2), ao Canil da Polícia Militar do Município de Araçatuba- SP por ceder os cães para o experimento e a Laine Margareth Gabas pelo auxílio técnico.

\section{REFERÊNCIAS}

ALBA-LOUREIRO, T.C.; MUNHOZ, C.D.; MARTINS, J.O. et al. Neutrophil function and metabolism in individuals with diabetes mellitus. Brazilian Journal of Medical and Biological Research, v.40, n.8, p.1037-1044, 2007.

BATISTA, J.J.; MARTINS, A.S.; MOROL, L. et al. Expressão gênica de caspases 3 e 8 em timo e baço de ratas recém-desmamadas e imunossuprimidas por glicocorticóide. Arquivo Brasileiro de Medicina Veterinária e Zootecnia, v.57, n.4, p.457-464, 2005.

BOSCO, A.M.; ALMEIDA, B.F.M.; PEREIRA,P.P. et al. High concentrations of glucose reduce the oxidative metabolism of dog neutrophils in vitro. BMC Veterinary Research, V.9, n.24, p.1-6, 2013.

BROWNLEE, M. Biochemistry and molecular cell biology of diabetic complications. Nature, v.13, n.14, p.813-820, 2001.

CAMPOS, P.P; VASCONCELOS, A.C; MELO, M.M. Apoptose no placentomo de cabras gestantes intoxicadas experimentalmente com cipó-preto - Tetrapterys multiglandulosa.

Arquivo Brasileiro Medicina Veterinária e Zootecnia, v.56, n.1, p.19-24, 2004.

COHEN, G; RUDNIKI, M; WALTER, F.et al. Glucose-modified proteins modulate essential functions and apoptosis of polymorphonuclear leukocytes. Journal American Society Nephrology, v.12, p. 1264-1271, 2001.

FEITOSA, F.L. Semiologia Veterinária: a arte do diagnóstico. São Paulo - SP: ROCA, cap.4, 2008. p. 77-102.

GLOWACKA, E.; BANASIK, M.; LEWKOWICZ, P. et al. The effect of LPS on neutrophils from patients with high risk of type 1 diabetes mellitus in relation to IL-8, IL-10 and IL-12 production and apoptosis in vitro. Scandinavian Journal of Immunology, v.55, p.210-217, 2002.

GUPTA, S.; KOIRALA, J.; KHARDORI, R.et al. Infections in diabetes mellitus and hyperglycemia. Infections disease clinics of North America, v.21, p.617-638, 2007.

KANEKO, J. J.; HARVEY, J. W.; BRUSS, M. L. Clinical biochemestry of domestic Animals, 6.ed. San Diego: Academic Press, 2008, 916p.

MAJEWSKA, E.; SULOWSKA, Z.; BAJ, Z. Spontaneus apoptosis of neutrophils in whole blood and its relation to apoptosis gene proteins. Scandinavian Journal Immunology, v 52, p.496-501, 2000. 
MCMANUS, L. M.; BLOODWORTH, R. C.;

PRIHODA, T. J. et al. Agonist-dependent failure of neutrophil function in diabetes correlates with extent of hyperglycemia. Journal of Leukocyte Biology, v.70, n.3, p.395-404, 2001.

NAGAMI, K.; KAWASHIMA, Y.; KUNO, H. et al. In vitro cytotoxicity assay to screen compounds for apoptosis-inducing potential on lynfocytes and neutrophils. The Journal of Toxicological Sciences, v.27, n.3, p. 91-203, 2002.

NELSON, R.W.; COUTO, C.G. Medicina Interna de Pequenos Animais, $3^{\circ}$ ed. Rio de Janeiro - RJ: Guanabara Koogan, 2006, 1325p.

OGUMA, K.; KANO, R.; HASEGAWA, A. In vitro study of neutrophil apoptosis in dogs. Veterinary Immunology and Immunopathology, v.76, p.157-162, 2000.

PITHON-CURI, T.C.; SCHUMACHER, R.I; FREITAS, J.J.S; et al. Glutamine delays spontaneous apoptosis in neutrophil. American Journal of Physiology ,v.284, p.355-1361, 2003.

SELA, S.; SHURTZ-SWIRSKI, R.; COHENMAZOR, M. et al. Primed peripheral polymorphonuclear leukocyte: a culprit underlying chronic low-grade inflammation and systemic oxidative stress in chronic kidney disease. J. Am. Soc. Nephrol., v.16, p.24312438, 2005.

SQUIER, M.K.T.; SEHNERT, A.J.; COHEN, J.J. Apoptosis in leukocytes. Journal Leukocyte Biological, v.57, p.2-10, 1995.

TENNENBERG, S.D.; FINKENAUER, R.; DWIVEDI, A. Absence of lipopolysaccharideinduced inhibition of neutrophil apoptosis in patients with diabetes. Archives of Surgery, v.134, n.11, p.1229-1234, 1999.

TIZARD, I.R. Imunologia Vaterinária, $6^{a}$ ed. São Paulo - SP: Editora Roca, cap. 3, 2002.p. 19-27.

TURINA, M; MILLER, F.N.; TUCKER, C. et al. Effects of hyperglycemia, hyperinsulinemia, and hyperosmolarity on neutrophil apoptosis.

Surgical infections, v.7, n.2, p.111-121, 2006.

WHYTE, M.K; MEAGHER, L.C.; MacDERMOT, J. et al. Impairment of function in aging neutrophils is associated with apoptosis. Journal of Immunology, v.150, n.11, p.51245134, 1993.

ZAR, J.H. Bioestatistical analysis. $2^{\mathrm{a}}$ ed. Englewood Cliffs: Pretice Hall,1984. 718p. 